19 Revue d'histoire du XIXe siècle

Société d'histoire de la révolution de 1848 et des

révolutions du XIXe siècle

$1 \mid 1985$

Varia

\title{
La serie CC aux archives nationales : cour des Pairs. Procès politiques
}

Jeannine Charon-Bordas

\section{(2) OpenEdition \\ Journals}

Édition électronique

URL : http://journals.openedition.org/rh19/5

DOI : $10.4000 /$ rh 19.5

ISSN : $1777-5329$

Éditeur

La Société de 1848

Édition imprimée

Date de publication : 1 juin 1985

ISSN : 1265-1354

Référence électronique

Jeannine Charon-Bordas, "La serie CC aux archives nationales : cour des Pairs. Procès politiques », Revue d'histoire du XIXe siècle [En ligne], 1 | 1985, mis en ligne le 28 octobre 2002, consulté le 30 avril 2019. URL : http://journals.openedition.org/rh19/5 ; DOI : 10.4000/rh19.5

Ce document a été généré automatiquement le 30 avril 2019.

Tous droits réservés 


\title{
La serie CC aux archives nationales : cour des Pairs. Procès politiques
}

\author{
Jeannine Charon-Bordas
}

La série CC conserve les archives issues du Sénat du Premier Empire et de la Chambre des pairs qui a siégé entre 1815 et 1848. Inséré au milieu de la série CC, on trouve un groupe documentaire de plus de trois cents articles relatif à l'activité de la Chambre Haute siégeant en cour de justice entre 1815 et 1847. Les procès jugés par la Cour des pairs sont naturellement hétérogènes, mais un certain nombre d'entre eux peut intéresser l'historien des mouvements sociaux au XIX ${ }^{\mathrm{e}}$ siècle.

Les procès jugés par la Cour des pairs sont connus tout d'abord par les publications faites par la Cour elle-même au moment des procès. Cependant le fonds conservé dans la série CC a gardé sa valeur propre ; la Cour n'a évidemment pas publié tous les documents réunis pour l'instruction; parfois même, elle a volontairement passé sous silence certains documents, ce qui rend les pièces originales plus précieuses. En outre, à l'occasion des arrestations opérées, les commissaires de police ont procédé à de nombreuses perquisitions et à des saisies de pièces à conviction, de correspondances et d'écrits divers, pièces qui n'ont été qu'exceptionnellement reproduites dans les publications de la Cour. Ces considérations et l'importance numérique du groupe documentaire CC 499 à 812 resté jusqu'ici sans inventaire même manuscrit, nous ont amené à établir un inventaire de ces articles, inventaire dont la publication est en cours ${ }^{1}$. Le fonds a été divisé en trois tranches chronologiques, la première correspondant à la période de la Restauration, dont les procès ne présentent guère d'intérêt dans l'optique de cette réunion; la seconde correspondant aux années 1830-1835 englobe l'énorme procès des insurrections d'avril 1834, le plus important de la série CC ; la troisième, enfin, correspond aux années 1835-1847, années d'activité intense de la Cour, pendant lesquelles prédominent les attentats contre le roi, mais où l'on trouve aussi le procès des journées insurrectionnelles des 12 et 13 mai 1839.

Cent seize articles renferment les dossiers des inculpés des insurrections d'avril 1834, les papiers saisis sur eux, à leur domicile ou dans leurs cellules de prison, les archives des associations ou des organes de presse poursuivis, les interrogatoires de ces mêmes 
inculpés, les dépositions des témoins, les rapports des autorités judiciaires et militaires, les enquêtes de police et les pièces de procédure.

On peut donc estimer que les dossiers de ce procès constituent une source de premier ordre pour la connaissance des protagonistes connus ou obscurs des émeutes de 1834. Les dossiers des inculpés sont évidemment très inégaux, mais beaucoup d'entre eux présentent un réel intérêt pour plusieurs raisons : d'abord, d'après une procédure largement suivie dans tous les procès jugés par la Cour des pairs, les autorités policières et les magistrats ont communiqué aux pairs les pièces relatives aux poursuites ou aux condamnations antérieures des inculpés, permettant ainsi de remonter dans leur passé (par exemple, pour les inculpés de Lyon en 1834, des documents relatifs à leur rôle dans les événements de 1831 ; le dossier Caussidière réunit les deux affaires du 21 février et d'avril 1834 à Saint-Étienne dans lesquelles Caussidière se trouve impliqué avec Nicot, etc. ) ; ensuite, les pièces saisies jointes en général aux dossiers des inculpés constituent d'authentiques archives du monde ouvrier (correspondance politique ou familiale, écrits divers, chansons et poèmes, règles de versification, tables pour coder la correspondance, pièces imprimées, petites brochures, pamphlets, libelles, chansonniers, publications à un sou ou à deux sous très répandues, statuts, listes de membres, comptes des associations, archives des journaux, essentiellement lyonnais et parisiens. En dernier lieu, il faut noter que les auteurs du Dictionnaire biographique du mouvement ouvrier français n'ont pas utilisé les dossiers de la série CC ni même les publications de la Cour des pairs, du moins pour les insurrections de 1834 ; par la suite, ils ont eu recours au moins aux publications. Il faut ici ouvrir une parenthèse sur le traitement subi par les archives des procès politiques après leur versement aux Archives nationales à la suite de la Révolution de 1848. Dans les années qui ont suivi ce versement, une partie des pièces à conviction et les objets saisis ont été distraits de leur série d'origine et placés dans les collections du Musée de l'Histoire de France, mesure qui se conçoit fort bien pour des objets (fusils, drapeaux, etc.) difficilement insérables dans une série de documents d'archives; mais, à cette occasion, on a également distrait de la série CC des papiers saisis sur les inculpés, manuscrits ou imprimés, dont la nature ne diffère pas des papiers restés dans les dossiers et qui auraient dû y être conservés. Pour remédier à cette mutilation bien inutile du fonds, nous avons ajouté en appendice au tome III de l'inventaire en cours, un inventaire analytique de tout ce qui est conservé dans les collections du Musée de l'Histoire de France et qui intéresse les procès politiques ; cela permettra de connaître l'existence de ces pièces qui restait jusqu'ici parfaitement ignorée.

Le tome 3 de l'inventaire des procès politiques comprend les douze affaires jugées entre 1835 et 1847, au nombre desquelles figurent sept attentats contre la personne du roi, affaires bien connues et qu'on pourrait croire sans intérêt. Cependant, il ne faut pas oublier que ces attentats ont été l'occasion d'enquêtes policières, d'arrestations, de perquisitions et de saisies de papiers dans les rangs de l'opposition républicaine pourtant décimée après la répression de 1834, de recherches concernant les sociétés secrètes qui se sont formées après le vote de la loi sur les associations, de poursuites contre la presse d'opposition. Il faut aussi noter que les fonds de la police (sous-série F7) font défaut pour toute cette période et que les dossiers de la série $\mathrm{CC}$ renferment des liasses de pièces confidentielles émanant souvent du cabinet du préfet de police, des notes de police sur les réunions des membres des sociétés secrètes, etc. que l'on ne peut évidemment plus trouver dans le fonds de la police. Ainsi, après l'attentat de Fieschi, les services de police enquêtèrent et procédèrent à des arrestations dans les rangs républicains (et aussi légitimistes) et impliquèrent un certain nombre de directeurs de journaux (Desnoyers, 
Raspail, Carrel) ; des papiers furent saisis au bureau du Charivari. Lors du procès de Meunier, furent annexées des copies de documents relatifs aux sociétés secrètes. Plus intéressants se révèlent les dossiers du procès de Darmès en raison de la personnalité de ce dernier qui le rattache à la fois à l'Église française de l'abbé Chatel et aux sociétés communistes ; une masse importante de papiers fut saisie, en particulier au domicile d'Henri Stévenot et également à Rouen, chez Charles Noiret "gloire ouvrière méconnue sinon inconnue", selon le Dictionnaire du mouvement ouvrier. Enfin, l'attentat commis par Quenisset n'aurait guère d'intérêt si on n'avait pas jugé à propos d'y rattacher le rédacteur du Journal du Peuple, d'ailleurs complètement étranger à l'attentat. Des perquisitions furent opérées au domicile de Dupoty et dans les bureaux du journal et des papiers furent saisis. En outre, un article renferme des pièces extraites de la procédure instruite devant le tribunal civil de la Seine contre les nommés Charavay et autres, fondateurs du journal L'Humanitaire, arrêtés précisément la veille de l'attentat alors qu'ils semblaient diriger une émeute qui avait éclaté place du Châtelet.

Les journées insurrectionnelles des 12 et 13 mai 1839 visaient au renversement du gouvernement monarchique et à l'instauration d'une république sociale; les noms de Blanqui, Barbès et Martin-Bernard apparaissent à cette occasion dans le fonds de la Cour des pairs. Les archives de ce procès réunissent 750 dossiers d'inculpés dont beaucoup d'ailleurs, bénéficièrent d'un non-lieu, dossiers qui sont une source intéressante pour l'étude des sociétés secrètes. Société des Saisons qui avait succédé à la Société des Familles, sociétés plus fugitives comme la Société des Vengeurs.

Comme on l'a déjà fait observer pour les tentatives dirigées contre la vie du roi, l'intérêt des dossiers conservés dans la série CC se situe un peu en dehors des affaires proprement dites, par ailleurs bien connues, dans les rapports de police, les liasses de pièces confidentielles qui suppléent aux lacunes de la sous-série $F 7$, et dans les pièces saisies qui nous livrent les papiers des inculpés. Les pièces saisies à l'occasion du procès des journées de 1839 sont particulièrement importantes : papiers saisis au domicile de Blanqui (parmi lesquels une liasse relative à son inculpation dans l'affaire des poudres de la rue de l'Oursine), au domicile du marchand de vin Charles chez lequel se tenaient des réunions de sociétés secrètes, et de nouveau au domicile du compositeur d'imprimerie Henri Stévenot.

Nous ne voudrions pas conclure sans signaler l'existence d'un versement de la $12^{\mathrm{e}}$ Chambre de la Cour d'appel de Paris, effectué en 1975 ; il concerne les archives des Hautes Cours qui ont siégé à Bourges et à Versailles en 1849 pour juger les inculpés des journées des 15 mai 1848 et 13 juin 1849. Ce versement a fait l'objet d'un classement et d'un inventaire sommaire établi par Madame Bertho, alors stagiaire à la Section Moderne ; il serait souhaitable de voir cet inventaire repris et développé et mis enfin à la disposition du public dans la Salle des Inventaires des Archives. Pour te moment, cet inventaire du groupe documentaire coté W 568 à 586 est consultable dans mon bureau. Bien que les hautes cours de la Seconde République ne se rattachent en rien sur le plan juridique à la Cour des pairs, l'ensemble des fonds de ces juridictions constitue une source historique importante pour toute la période couvrant la monarchie de Juillet et la Seconde République.

La série $\mathrm{C}$ (fonds des assemblées nationales) conserve sous les cotes $\mathrm{C} 3103$ à 3128 les papiers de la Commission des Grâces instituée le 10 juillet 1871 pour examiner les recours en grâce formés par les insurgés de la Commune. Le recours en grâce était la dernière procédure que pouvait engager un condamné après avoir formé un pourvoi en revision et un pourvoi en cassation ; quelquefois, le condamné n'avait formé que le pourvoi en 
révision, quelquefois, il n'avait formé aucun pourvoi et s'adressait à la Commission directement pour obtenir une commutation de peine ou une grâce pure et simple. La Commission n'examinait naturellement que les dossiers des condamnés ayant formé un recours (eux-mêmes ou leur famille) ; une dérogation fut cependant prévue pour les condamnés à la peine capitale dont les dossiers furent examinés d'office par la Commission.

Ce groupe documentaire a au moins le mérite de nous être parvenu dans son intégrité sans avoir subi comme les dossiers de grâce conservés dans la sous-série BB 24 des tris fâcheux. 6530 rapports, dont certains relatifs à plusieurs accusés, adressés par le Garde des Sceaux avec un avis de l'autorité militaire ajouté à la suite du rapport, nous sont parvenus classés dans un ordre alphabétique quelque peu imparfait (surtout dans l'ordre des prénoms en cas d'homonymie) mais aisément repérables.

Ces rapports recoupent évidemment d'autres sources, bien connues et largement exploitées. Pour la Section Moderne, il faut citer en premier lieu les fichiers des condamnés de la Commune cotés BB 27724 à 871 . Sans doute aurait-on pu craindre que les rapports conservés dans la série $\mathrm{C}$--fassent double emploi avec ceux de la sous-série BB 24, mais les sondages effectués laissent apparaître qu'il n'en est rien.

Trois registres cotés conservés dans l'article $\mathrm{C} 3103$ renferment les procès-verbaux des séances de la Commission qui a siégé d'août 1871 au 7 mars 1876. Un article du règlement qui régissait les travaux de la Commission des Grâces stipulait que les résultats des votes des membres devaient être tenus secrets et que les noms des rapporteurs et des divers orateurs ne devaient pas être mentionnés. Il eût été intéressant, pourtant, de connaître les opinions des députés formant la Commission, tous issus de la province, qui eurent à juger les protagonistes d'une insurrection parisienne. Trente trois registres cotés C 3124 à 3128 renferment les avis donnés par la Commission sur les recours en grâce.

Un inventaire analytique manuscrit a été établi pour ce groupe documentaire.

\section{NOTES}

${ }^{1}$ CHARON-BORDAS Jeannine, Conservateur aux Arch. nat. Cour des Pairs : procès politiques. Avant-propos de Jean Favier. Paris, Arch. nat. tome 1., La Restauration : inventaire des articles CC 499 à 545, 1982, 106 p. ; tome 2., La monarchie de Juillet : inventaire des articles CC 546 à 670, 1983, 259 p. ; tome 3., A paraître en 1984. Diffusion : La Documentation Française, et Arch. nat.

\section{INDEX}

Mots-clés : Archives, Pairs, Répression, Procès 